\title{
The Remnants of Intergalactic Supernovae
}

\section{Citation}

Maoz, Dan, Eli Waxman, and Abraham Loeb. 2005. "The Remnants of Intergalactic Supernovae." The Astrophysical Journal 632 (2): 847-53. https://doi.org/10.1086/433174.

\section{Permanent link}

http://nrs.harvard.edu/urn-3:HUL.InstRepos:41393446

\section{Terms of Use}

This article was downloaded from Harvard University's DASH repository, and is made available under the terms and conditions applicable to Other Posted Material, as set forth at http:// nrs.harvard.edu/urn-3:HUL.InstRepos:dash.current.terms-of-use\#LAA

\section{Share Your Story}

The Harvard community has made this article openly available.

Please share how this access benefits you. Submit a story.

Accessibility 


\title{
THE REMNANTS OF INTERGALACTIC SUPERNOVAE
}

\author{
Dan Maoz, ${ }^{1}$ Eli Waxman, ${ }^{2}$ and Abraham Loeb ${ }^{3}$ \\ Received 2005 May 1; accepted 2005 June 19
}

\begin{abstract}
Intergalactic Type Ia supernovae ( $\mathrm{SNe}$ ) have been discovered recently in rich galaxy clusters. These $\mathrm{SNe}$ are likely the descendants of an intergalactic stellar population, which has been discovered in recent years through a variety of tracers. We estimate the observational signatures of the associated SN remnants (SNRs) in the unusual intracluster medium (ICM) environment. We find that if Type Ia SNe still have a circumstellar medium (CSM) at the time of explosion, then their remnants are visible in the optical for $\sim 10^{2}-10^{3} \mathrm{yr}$, with properties similar to young galactic SNRs. In contrast with galactic SNRs, in which the ejecta from the explosion interacts with the interstellar medium (ISM), intracluster SNRs become undetectable in the optical band once their ejecta passes beyond the CSM and enters the hot and tenuous ICM. If Type Ia SNe have a CSM, there should be $\sim 150$ young SNRs in the nearby Virgo Cluster, with an $\mathrm{H} \alpha$ luminosity of $\sim 10^{35} \mathrm{ergs} \mathrm{s}^{-1}$ and an angular size of $\sim 0$ ". 1 . We investigate the possibility that members of this SNR population may have recently been detected but incorrectly identified as intergalactic $\mathrm{H}$ II regions. Alternatively, if optical intergalactic SNRs do not exist in Virgo, this will constitute evidence that Type Ia SNe are devoid of a CSM, with implications for progenitor scenarios. Regardless of the presence of a CSM, about 10 older SNRs per square degree should be detectable in Virgo in the radio band, with fluxes of order $0.1 \mathrm{mJy}$ at $1 \mathrm{GHz}$. Their angular sizes $\left(\sim 1^{\prime \prime}\right)$, morphologies, and lack of optical association with distant galaxies can distinguish them from the much more numerous background population. Their detection would provide an accurate measurement of the intracluster SN rate. Deep pointed observations toward the site of SN 1980I, a possibly intergalactic Type Ia event in Virgo, could test for the existence of a CSM by comparison to our predictions for the early-time development of intergalactic SNRs.
\end{abstract}

Subject headings: galaxies: clusters: general — supernova remnants — supernovae: general

\section{INTRODUCTION}

Studies of nearby clusters have revealed a population of intergalactic stars. Already noticed by Zwicky (1951) as excess starlight between the galaxies in the core of the Coma Cluster, this diffuse stellar emission has been confirmed and quantified in recent deep images of Coma (Gregg \& West 1998; Trentham \& Mobasher 1998; Feldmeier et al. 2002), in other nearby clusters (Calcáneo-Roldán et al. 2000), and in stacked images of redshift $z \sim 0.25$ clusters from the Sloan Digital Sky Survey (Zibetti et al. 2005). Intergalactic red giant stars have been detected in the Virgo Cluster (Ferguson et al. 1998; Durrell et al. 2002), and intergalactic planetary nebulae have been found in Virgo and Fornax (Arnaboldi et al. 1996; Theuns \& Warren 1997; Mendez et al. 1997; Ciardullo et al. 1998, 2002; Feldmeier et al. 1998). Some $10 \%-20 \%$ of the stars in galaxy clusters are in the intergalactic component. This population is believed to have been stripped off the cluster galaxies through tidal disruption by other galaxies and by the cluster potential as a whole (Dubinski et al. 1996; Moore et al. 1996; Korchagin et al. 2001).

In the course of a survey for supernovae (SNe) in rich galaxy clusters at redshifts $0.08<z<0.2$, Gal-Yam et al. (2003) recently discovered two Type Ia SNe at the redshifts of their respective clusters, but spatially and kinematically distinct from any galaxy in the cluster. The two events, constituting $2 / 7$ of the cluster $\mathrm{SNe}$ found in the survey, had no detectable host galaxy, even in deep images taken by the Keck 10 m telescope. Gal-Yam

\footnotetext{
${ }^{1}$ School of Physics and Astronomy, Tel Aviv University, Tel Aviv 69978, Israel; dani@wise.tau.ac.il.

2 Physics Department, Weizmann Institute, Rehovot 70100, Israel; waxman@ wicc.weizmann.ac.il.

3 Astronomy Department, Harvard University, 60 Garden Street, Cambridge, MA 02138; and Einstein Minerva Center, Weizmann Institute, Rehovot 70100, Israel; aloeb@cfa.harvard.edu.
}

et al. argued, based on the galaxy luminosity function of clusters, that dwarf galaxies below the detection limit and contributing only $\sim 10^{-3}$ of the cluster stellar luminosity could not plausibly be the hosts of the two SNe. Accounting for the relative detection efficiencies of events within and outside galaxies, Gal-Yam et al. estimated that $21_{-14}^{+18}$ percent of the SN Ia parent stellar population in clusters is intergalactic. This fraction is consistent with the intergalactic stellar fraction found by other tracers.

Quantifying the properties of the intergalactic stellar population via its different tracers is important for understanding galaxy interactions and evolution in dense environments. $\mathrm{SNe}$ are particularly useful because, as opposed to other tracers, they can be seen out to clusters at large look-back times and can thus reveal the history of the galaxy evolution process.

Supernovae are brief optical events. Supernova remnants (SNRs), however, exist for thousands of years and are detectable over a wide range of wavelengths. The intergalactic SN population in clusters could hence potentially be detected and characterized by means of the SNRs it leaves behind. In this paper, we predict the observational signatures of SNRs in the unusual intracluster medium (ICM) environment. We show that the SN ejecta is visible via its interaction with the surrounding circumstellar medium (CSM), if the latter exists, over a timescale of $\sim 10^{2}-10^{3} \mathrm{yr}$. In galactic environments, after traversing the CSM, the ejecta interacts with a galaxy's interstellar medium (ISM) and the SNR remains visible for $10^{4} \mathrm{yr}$ or more. However, in cluster environments, once the ejecta traverses the CSM and enters into the hot and tenuous intracluster medium (ICM), the SNR emission fades. We will thus argue that the emission from the remnants of intergalactic Type Ia SNe is detectable only if they are surrounded by a dense CSM (implying progenitors with a giantstar companion that had a high mass-loss rate; see review by Branch et al. 1995) and if the remnants are not much older than a thousand years. A CSM has been detected in only one Type Ia 
SN, 2002ic, where hydrogen lines have appeared in the latetime spectra of the explosion (Hamuy et al. 2003). However, it is unclear whether this single event is representative of the physical conditions of most SNe Ia which (by definition) have no signs of hydrogen in their spectra. Thus, detection of intergalactic SN Ia remnants could demonstrate the existence of a CSM around Type Ia SN progenitors.

In $\S 2$ we use some simple physical arguments to derive the main observed features of SNRs and their dependence on progenitor properties and on the peculiar ICM environment. In $\S 3$ we review the characteristics of the unresolved intergalactic emission-line objects recently discovered in nearby clusters and groups and discuss whether some or all of these objects could be intergalactic SNRs, rather than $\mathrm{H}$ II regions. In $\S 4$ we examine whether radio observations could detect the intergalactic SNR population. We summarize our results in $\S 5$.

\section{THE APPEARANCE OF A SUPERNOVA REMNANT IN THE INTRACLUSTER MEDIUM}

\subsection{Wind Dynamics}

Here we construct a simple physical model of SNRs that reproduces their main observed features and allows the prediction of the properties of intergalactic SNRs. For a detailed treatment of the physics of SNRs, see, e.g., McCray \& Wang (1996) or Truelove \& McKee (1999).

We consider a Type Ia SN explosion due to a white dwarf that exceeds the Chandrasekhar mass through accretion of wind material from a giant-star companion. The giant is assumed to have a mass-loss rate of $\dot{M}=10^{-6} \dot{M}_{-6} M_{\odot} \mathrm{yr}^{-1}$, which creates a CSM with which the $\mathrm{SN}$ ejecta eventually collides. Only later does the ejecta reach the external medium, be it the ISM in the case of normal galactic $\mathrm{SNe}$ or the ICM for the intracluster $\mathrm{SNe}$ under discussion.

First we consider the case where the binary star system is at rest relative to an ambient ICM with a pressure $p_{\mathrm{ICM}}$. In this case, the entire mass lost by the giant star accumulates in a shell around the binary. This case applies when the speed of the binary system relative to the ICM, $v_{\text {star }}$, is much smaller than the giant star's wind speed, $v_{w}=10^{1.5} v_{w, 1.5} \mathrm{~km} \mathrm{~s}^{-1}$.

The outgoing wind acts as a piston and generates a forward shock in the external medium (e.g., Parker 1963). Since the wind velocity is typically much smaller than the sound speed of the surrounding ICM, the forward shock is weak. The interaction with the ICM sends a reverse shock back into the cold wind material. The shell of material trapped between the forward and reverse shocks includes wind material separated from shocked ICM material by a contact discontinuity. If this shell is moving forward with a velocity $v$, then the reverse shock moves backward relative to the unperturbed wind at a speed of

$$
v_{s}=\frac{(\gamma+1)}{2}\left(v_{w}-v\right)
$$

where $\gamma=5 / 3$ is the adiabatic index of a monoatomic gas. The pressure in this shell satisfies

$$
p_{s}=\frac{2}{(\gamma+1)} \rho_{w} v_{s}^{2} \approx p_{\mathrm{ICM}}
$$

implying a reverse-shock speed relative to the ICM of

$$
v_{\mathrm{rs}}=\left(v_{w}-v_{s}\right)=v_{w}\left[1-\sqrt{\frac{(\gamma+1) p_{\mathrm{ICM}}}{2 \rho_{w} v_{w}^{2}}}\right] .
$$

Here $\rho_{w} \propto R^{-\eta}$ is the density of the wind material, with $\eta=2$ if the star had a constant mass-loss rate. For such a density profile, the reverse shock turns into a standing shock in the observer's frame at the radius $R=R_{\mathrm{rs}}$ where $v_{\mathrm{rs}}=0$, namely $v_{w}=v_{s}$. Setting $v_{\text {rs }}=0$ in equation (3), we have

$R_{\mathrm{rs}}=\left[\frac{\dot{M} v_{w}}{2 \pi(\gamma+1) p_{\mathrm{ICM}}}\right]^{1 / 2}=0.6 \times 10^{18} \dot{M}_{-6}^{1 / 2} v_{w, 1.5}^{1 / 2} n_{-3}^{-1 / 2} T_{1}^{-1 / 2} \mathrm{~cm}$,

where $\rho_{w} \equiv n_{w} m_{p}=\dot{M} / 4 \pi R^{2} v_{w}$ is the mass density in the wind (with $m_{p}$ being the mean particle mass), $T_{\mathrm{ICM}}=10 T_{1} \mathrm{keV}$ is the ICM temperature, and $n_{\mathrm{ICM}}=10^{-3} n_{-3} \mathrm{~cm}^{-3}$ is the electron density in the ICM (with $p_{\mathrm{ICM}} \approx 2 n_{\mathrm{ICM}} k T_{\mathrm{ICM}}$ ). At this point the wind velocity is reduced to

$$
v=\left(\frac{\gamma-1}{\gamma+1}\right) v_{w}=\frac{1}{4} v_{w} .
$$

The density profile beyond $R_{\mathrm{rs}}$ can be derived by the following argument. Once a wind fluid element crosses the reverse shock, its entropy is conserved and its pressure is approximately time independent and equal to the ICM pressure. This implies that the density of the fluid is independent of time and radius at $R>R_{\mathrm{rs}}, \rho_{w}\left(R>R_{\mathrm{rs}}\right)=4 \dot{M} / 4 \pi R_{\mathrm{rs}}^{2} v_{w}=2(\gamma+1) p_{\mathrm{ICM}} / v_{w}^{2}$, i.e.,

$$
\rho_{w}\left(R>R_{\mathrm{rs}}\right)=9.2 m_{p} \frac{n_{-3} T_{1}}{v_{w, 1.5}^{2}} \mathrm{~cm}^{-3} .
$$

The radius-independent density implies a deceleration of the wind velocity beyond $R_{\mathrm{rs}}, v_{w}\left(R>R_{\mathrm{rs}}\right) \propto R^{-2}$. The outer radius of the wind is determined by mass conservation, $4 \pi R_{\max }^{3} \rho_{w}(R>$ $\left.R_{\mathrm{rs}}\right) / 3=\dot{M} \tau$,

$R_{\max }=\left(\frac{3}{4} R_{\mathrm{rs}}^{2} v_{w} \tau\right)^{1 / 3}=3.0 \times 10^{18}\left(\frac{\dot{M}_{-6} v_{w, 1.5}^{2} \tau_{6}}{n_{-3} T_{1}}\right)^{1 / 3} \mathrm{~cm}$.

Here $\tau=10^{6} \tau_{6} \mathrm{yr}$ is the duration of the period over which the wind is active before the SN explodes. For typical parameters, most of the wind material is trapped between this radius and the stationary radius of the reverse shock, $R_{\mathrm{rs}}$.

In the ICM of massive clusters, an intergalactic stellar binary will typically move at a high speed of $v_{\text {star }} \sim 10^{3} \mathrm{~km} \mathrm{~s}^{-1}$ relative to the ICM. Under these circumstances, the wind remnant will be stripped by the ram pressure of the ICM in its rest frame. We define an effective "ram pressure" speed $v_{\star}=\left(v_{*}^{2}+v_{\text {th }}^{2}\right)^{1 / 2}=$ $10^{3} v_{*, 3} \mathrm{~km} \mathrm{~s}^{-1}$, where $v_{\mathrm{th}}^{2}=p_{\text {ICM }} / \rho_{\text {ICM }}$ is the thermal speed of the ICM gas. The wind remnant (or equivalently, the CSM) will have the same structure as before, except that the ICM temperature $T$ is increased by a factor $\sim\left(v_{\star} / v_{\text {th }}\right)^{2}$ to $T_{*}=\left(1+v_{*}^{2} / v_{\text {th }}^{2}\right) T$. This leads to modification of the stalling radius of the reverse shock, $R_{\mathrm{rs}}$, of $R_{\mathrm{max}}$, and of $\rho_{w}\left(R>R_{\mathrm{rs}}\right)$. A bow shock structure will form, with these modified parameters describing its forward region. In the other directions, there will be larger radii and lower densities, but only by factors of order unity.

We assume that the CSM gas is neutral, both in front of and behind the reverse shock, based on the following arguments. First, we consider the effect of the reverse shock on the CSM gas that has passed through it. Since the pressure of this gas approximately equals the ICM pressure (eq. [2]), equation (6) implies that the post-reverse-shock CSM temperature is

$$
T_{\mathrm{CSM}} \approx 10^{4} K v_{w, 1.5}^{2}
$$


Thus, the relatively mild reverse shock may ionize the gas at $R>R_{\mathrm{rs}}$ but will not heat it excessively. If the gas gets ionized, its recombination time will be

$$
t_{\mathrm{rec}} \approx 1.5 \times 10^{4} \mathrm{yr} v_{w, 1.5}^{2} n_{-3}^{-1} T_{1}^{-1},
$$

much shorter than the $\sim 10^{6} \mathrm{yr}$ duration of the wind phase. Thus, by the time the SN explodes, the CSM will have become neutral again. Second, the radiation from the ICM also cannot photoionize the CSM. The photoionization cross section of neutral hydrogen declines with increasing frequency as $\nu^{-3}$ above the ionization threshold, while the optically thin thermal bremsstrahlung flux from the ICM, per unit frequency, is independent of frequency for $h \nu \ll k T$. Thus, only photons with energy within a factor of 2 above $13.6 \mathrm{eV}$ contribute to ionizations. For the fiducial ICM parameters, $n_{-3}=1$ and $T_{1}=1$, and assuming a cluster core radius of $\sim 300 \mathrm{kpc}$, the bremsstrahlung impinging on the CSM surface has a photon flux of $\sim 4 \times 10^{3} \mathrm{~cm}^{-2} \mathrm{~s}^{-1}$ between 13.6 and $27.2 \mathrm{eV}$ (e.g., Rybicki \& Lightman 1979, pp. 159-162). Multiplying by the ionization cross section at $13.6 \mathrm{eV}, 6.3 \times 10^{-18} \mathrm{~cm}^{2}$, the ionization rate is $\sim 2 \times 10^{-14} \mathrm{~s}^{-1}$ per hydrogen atom. For the fiducial CSM density of $\sim 10 \mathrm{~cm}^{-3}$ (eq. [6]), the recombination rate is $\sim 2 \times 10^{-12} \mathrm{~s}^{-1}, 100$ times higher than the photoionization rate, and therefore the CSM gas will remain mostly neutral. Finally, the ICM gas particles cannot collisionally heat and ionize the CSM because magnetic fields keep their mean free path small, thus suppressing heat conduction. This situation is actually observed in the case of "cold fronts," clouds of relatively cool gas that maintain their lower temperature as they plunge through an X-ray cluster (e.g., Vikhlinin $\&$ Markevitch 2002). The magnetic fields also provide surface tension that prevents shearing of the cold gas clouds via the Kelvin-Helmholtz instability.

\subsection{Ejecta Dynamics and Line Emission}

Once the SN explodes, the fast SN ejecta expands and drives a strong shock into the CSM. By definition, the ejecta of Type Ia explosions have a low abundance of hydrogen. The $\mathrm{H} \alpha$ emission from their remnants originates from the neutral hydrogen intercepted by the ejecta's forward shock in the surrounding medium. The underlying physics of $\mathrm{H} \alpha$ emission at the young stage (when the shock is called a "nonradiative" shock and the SNR is called "Balmer dominated") is well understood (Raymond 1991). Of order one-tenth of all hydrogen atoms entering the shock produce an $\mathrm{H} \alpha$ photon before they get ionized, and roughly another tenth of the atoms do it by charge exchange with the hot electrons behind the shock. The first component generates a narrow line (since the emission occurs before the atoms get kicked by the shock), and the second results in a broad $\mathrm{H} \alpha$ component with a velocity width of order the shock speed.

The total $\mathrm{H} \alpha$ luminosity can be calculated from the radius of the forward shock, $R_{\mathrm{fs}}$,

$$
L_{\mathrm{H} \alpha}=4 \pi R_{\mathrm{fs}}^{2} \frac{d R_{\mathrm{fs}}}{d t}\left(\frac{\rho_{\mathrm{CSM}}}{m_{p}}\right) 0.2 h \nu_{\mathrm{H} \alpha}
$$

where $h \nu_{\mathrm{H} \alpha}=3 \times 10^{-12}$ ergs is the energy of an $\mathrm{H} \alpha$ photon. The CSM mass density at radii $R<R_{\mathrm{rs}}$ is given by $\rho_{\mathrm{CSM}}=$ $\dot{M} / 4 \pi R^{2} v_{w}$, yielding

$$
L_{\mathrm{H} \alpha}=\frac{\dot{R}_{\mathrm{fs}}}{v_{w}} \frac{\dot{M}}{m_{p}} 0.2 h \nu_{\mathrm{H} \alpha} .
$$

The forward-shock velocity may be estimated as follows. Ignoring, at first, the interaction of the SN ejecta with the CSM, the time-dependent ejecta velocity profile is given, after significant expansion of the ejecta, by $v=R / t$. This velocity profile describes "free expansion" (expansion into vacuum of a pressureless fluid), in which the velocity of each fluid element is independent of time. The density profile of such a flow is self-similar, $\rho(R, t) \propto g(R / t) t^{-3}=g(v) t^{-3}$. Numerical models of SN Ia explosions yield $g(v)=\exp \left(-v / v_{\mathrm{ej}}\right)$ (e.g., Höflich \& Khokhlov 1996; Dwarkadas \& Chevalier 1998). The characteristic ejecta speed $v_{\mathrm{ej}}$ defines the ratio of the ejecta kinetic energy $E$ to mass $M$,

$$
\frac{E}{M}=\frac{1}{2} \frac{\int d R R^{2} \rho v^{2}}{\int d R R^{2} \rho}=\frac{\int d v v^{4} g(v)}{2 \int d v v^{2} g(v)} .
$$

For $g(v)=\exp \left(-v / v_{\mathrm{ej}}\right)$ we have

$$
v_{\mathrm{ej}}=\left(\frac{E}{6 M_{\mathrm{ej}}}\right)^{1 / 2}=10^{3.5}\left(\frac{E_{51}}{M_{\mathrm{ej}, 0}}\right)^{1 / 2} \mathrm{~km} \mathrm{~s}^{-1},
$$

where $E=10^{51} E_{51}$ ergs and $M_{\mathrm{ej}}=10^{0} M_{\mathrm{ej}, 0} M_{\odot}$ (see, e.g., Truelove \& McKee 1999, for a summary of the values of these parameters in historical SNRs). The fraction of the ejecta mass that has a velocity in excess of $v$ is determined by the algebraic relation

$$
\frac{M(>v)}{M_{\mathrm{ej}}}=\frac{1}{2} \int_{v / v_{\mathrm{ej}}} d x x^{2} e^{-x}=\left[1+\left(1+\frac{1}{2} \frac{v}{v_{\mathrm{ej}}}\right) \frac{v}{v_{\mathrm{ej}}}\right] e^{-v / v_{\mathrm{ej}}} .
$$

The part of the ejecta with velocity $>v$ starts decelerating once it has propagated to a radius out to which the CSM mass is comparable to $M(>v)$. As long as $R_{\mathrm{fs}}<R_{\mathrm{rs}}$, the time at which this deceleration occurs is given by $t=M(>v) v_{w} / \dot{M} v$. Thus, the velocity of the "piston" driving the shock into the surrounding CSM is given as a function of time by

$$
t=\frac{M_{\mathrm{ej}} v_{w}}{\dot{M} v_{\mathrm{ej}}}\left[\frac{v_{\mathrm{ej}}}{v}+\left(1+\frac{1}{2} \frac{v}{v_{\mathrm{ej}}}\right)\right] e^{-v / v_{\mathrm{ej}}} .
$$

Defining a characteristic time for deceleration

$$
t_{\mathrm{ej}} \equiv \frac{M_{\mathrm{ej}} v_{w}}{\dot{M} v_{\mathrm{ej}}}=1.1 \times 10^{4} \frac{M_{\mathrm{ej}, 0}^{3 / 2} v_{w, 1.5}}{\dot{M}_{-6} E_{51}^{1 / 2}} \mathrm{yr},
$$

the solution of equation (15) for $t<t_{\mathrm{ej}}$ is

$$
v \approx 1.1 \ln \left(2 t_{\mathrm{ej}} / t\right) v_{\mathrm{ej}} .
$$

Equations (15) and (16) hold for $R_{\mathrm{fs}}<R_{\mathrm{rs}}$. The shock first reaches this radius at $t=t_{\mathrm{rs}}$, given by

$$
t_{\mathrm{rs}} \ln \frac{2 t_{\mathrm{ej}}}{t_{\mathrm{rs}}} \equiv \frac{R_{\mathrm{rs}}}{1.1 v_{\mathrm{ej}}}=58\left(\frac{\dot{M}_{-6} v_{w, 1.5} M_{\mathrm{ej}, 0}}{n_{-3} T_{* 1} E_{51}}\right)^{1 / 2} \mathrm{yr} .
$$

Since the forward shock is strong, its speed is simply related to the piston speed by $\left(d R_{\mathrm{fs}} / d t\right)=[(\gamma+1) / 2] v=(4 / 3) v$, and consequently

$$
L_{\mathrm{H} \alpha}=3.0 \times 10^{33} \frac{\dot{M}_{-6} E_{51}^{1 / 2}}{v_{w, 1.5} M_{\mathrm{ej}, 0}^{1 / 2}} \ln \frac{2 t_{\mathrm{ej}}}{t} \mathrm{ergs} \mathrm{s}^{-1}
$$

for $t<t_{\mathrm{rs}}$. 
For $t>t_{\mathrm{rs}}, R_{\mathrm{fs}}>R_{\mathrm{rs}}$, the piston velocity and time are related by $M(>v)=4 \pi(v t)^{3} \rho_{w}\left(R>R_{\mathrm{rs}}\right) / 3=(4 / 3) \dot{M}(v t)^{3} / R_{\mathrm{rs}}^{2} v_{w}$, i.e., by

$$
\left(\frac{t}{t_{\mathrm{ej}}^{\prime}}\right)^{3}=\left[\left(\frac{v_{\mathrm{ej}}}{v}\right)^{3}+\left(1+\frac{1}{2} \frac{v}{v_{\mathrm{ej}}}\right)\left(\frac{v_{\mathrm{ej}}}{v}\right)^{2}\right] e^{-v / v_{\mathrm{ej}}},
$$

with

$$
t_{\mathrm{ej}}^{\prime} \equiv\left(\frac{3 M_{\mathrm{ej}} v_{w} R_{\mathrm{rs}}^{2}}{4 \dot{M} v_{\mathrm{ej}}^{3}}\right)^{1 / 3}=360\left(\frac{M_{\mathrm{ej}, 0}^{5 / 2} v_{w, 1.5}^{2}}{E_{51}^{3 / 2} n_{-3} T_{* 1}}\right)^{1 / 3} \mathrm{yr} .
$$

At $t \leq t_{\mathrm{ej}}^{\prime}$ we have $v \approx v_{\mathrm{ej}}$. Here we neglect the logarithmic corrections to $v$ implied by equation (20), since for a uniform $\rho_{\mathrm{CSM}}$ the time dependence of $L_{\mathrm{H} \alpha}$ is given by $L_{\mathrm{H} \alpha} \propto R_{\mathrm{fs}}^{2} v \rho_{\mathrm{CSM}} \propto v t^{2}$, and the logarithmic evolution of $v$ is not important. We therefore obtain

$$
L_{\mathrm{H} \alpha}=2.8 \times 10^{35} M_{\mathrm{ej}, 0}^{1 / 6} E_{51}^{1 / 2}\left(\frac{n_{-3} T_{* 1}}{v_{w, 1.5}^{2}}\right)^{1 / 3}\left(\frac{t}{t_{\mathrm{ej}}^{\prime}}\right)^{\beta} \operatorname{ergs~s}^{-1}
$$

where $\beta=2$ for $R_{\mathrm{rs}} / v_{\mathrm{ej}}<t<t_{\mathrm{ej}}^{\prime}$ and $\beta=\frac{1}{5}$ for $t_{\mathrm{ej}}^{\prime}<t$. At $t_{\mathrm{ej}}^{\prime}<t$, equation (20) [which implies $v \approx v_{\mathrm{ej}}\left(t_{\mathrm{ej}}^{\prime} / t\right)$ ] no longer holds, and the shock approaches the self-similar Sedov-von NeumannTaylor regime with $R_{\mathrm{fs}} \propto t^{2 / 5}$.

Equation (22) holds up to the time where the ejecta traverses the CSM and enters the ICM. For $v_{\mathrm{ej}} t_{\mathrm{ej}}^{\prime}>R_{\max }$ the ejecta suffers little deceleration prior to crossing the CSM, and hence the crossing time is given by $t_{\mathrm{CSM}} \sim R_{\max } / v_{\mathrm{ej}}$. For $v_{\mathrm{ej}} t_{\mathrm{ej}}^{\prime}<R_{\max }$, the ejecta enters the self-similar deceleration phase before crossing the $\mathrm{CSM}$ and $t_{\mathrm{CSM}} \sim\left(R_{\max } / v_{\mathrm{ej}}\right)\left(R_{\max } / v_{\mathrm{ej}} t_{\mathrm{ej}}^{\prime}\right)^{3 / 2}$. Since $v_{\mathrm{ej}} t_{\mathrm{ej}}^{\prime} / R_{\max }=$ $\left(M_{\mathrm{ej}} / \dot{M} \tau\right)^{1 / 3}$, we find

$$
\begin{gathered}
t_{\mathrm{CSM}} \sim \frac{R_{\mathrm{max}}}{v_{\mathrm{ej}}} \max \left[1,\left(\frac{\dot{M} \tau}{M_{\mathrm{ej}}}\right)^{1 / 2}\right] \\
=360\left(\frac{\dot{M}_{-6} v_{w, 1.5}^{2} \tau_{6}}{n_{-3} T_{* 1}}\right)^{1 / 3}\left(\frac{E_{51}}{M_{\mathrm{ej}, 0}}\right)^{-1 / 2} \max \left[1,\left(\frac{\dot{M} \tau}{M_{\mathrm{ej}}}\right)^{1 / 2}\right] \mathrm{yr} .
\end{gathered}
$$

This time may change by a factor of order unity, depending on the binary speed $v_{\text {star }}$, and one could average over a Maxwellian distribution of possible stellar velocities to examine the statistics of SN events with different durations. Depending on the giant's wind speed, the duration of mass loss, and the ejecta velocity, the time to traverse the CSM may vary in the range $\sim 10^{2}-10^{3} \mathrm{yr}$.

As long as the ejecta is interacting with the CSM, an intergalactic SNR is thus similar to a galactic SNR at this stage of its development. We can therefore test the applicability of our simple model by comparing its predictions to the observed properties of young ( $\lesssim 1000 \mathrm{yr}$ ) Type Ia SNRs in the Milky Way and in nearby galaxies. The remnants of the historical SNe of 1006 and of 1572 (Tycho) belong to this class. Observational estimates of sizes and luminosities for Galactic SNRs are complicated by uncertainties in distance and extinction, but this problem is being overcome by the detection of SNRs in nearby galaxies. In the Large Magellanic Cloud, the typical $\mathrm{H} \alpha$ luminosities of young, Balmer-dominated SNRs are of order $10^{34}-10^{35} \mathrm{ergs} \mathrm{s}^{-1}$ (Tuohy et al. 1982; Smith et al. 1991). This is comparable to our estimate in equation (22). Note that the typical pressures $n T$ of the ICM and the ISM are similar, and hence in view of the weak ( $\frac{1}{3}$ power) dependence on pressure, we expect similar luminosities at this stage from galactic SNRs and intergalactic SNRs exploding into a CSM.

In older (a few thousand to 20,000 years old) Galactic and nearby SNRs, the radii are typically $10-15 \mathrm{pc}$, and $\mathrm{H} \alpha$ luminosities are in the range of $10^{35-37} \mathrm{ergs} \mathrm{s}^{-1}$ (e.g., Blair \& Long 2004; Williams et al. 2004). However, at this stage the shocks, now called "radiative shocks," have slowed down considerably. Cooling is dominated by hydrogen recombination and by collisionally excited lines of low-ionization metals, especially in regions where the shock encounters denser clumps with shorter recombination times. In fact, optical surveys often distinguish SNRs from $\mathrm{H}$ II regions based on an emission-line ratio criterion of [S II] $\lambda \lambda 6717,6731 / \mathrm{H} \alpha>0.4$, but of course, this will select against young, often Balmer-dominated SNRs. It is possible that intergalactic SNRs can approach this stage of greater Balmer line luminosity and a metal-line cooling spectrum in regions of higher pressure in the ICM (e.g., near the cluster center) or if the preexplosion wind included clumps of dense material. More likely, however, the forward shock will reach $R_{\max }$ and enter the ICM when it is still fast and nonradiative. Since the ICM is fully ionized, the $\mathrm{H} \alpha$ emission is expected to diminish as soon as the ejecta's forward shock traverses the CSM. This is in contrast to SNRs in the ISM of galaxies, where the forward shock continues to intercept neutral atoms even beyond the CSM.

\subsection{Continuum Emission}

The collisionless forward shock of the SN remnant is expected to accelerate electrons to relativistic energies by the Fermi mechanism (e.g., Blandford \& Eichler 1987). In the presence of intracluster magnetic fields, the accelerated electrons are expected to emit nonthermal radio photons via the synchrotron process (see Chevalier \& Raymond 1978 and references therein). Let us first consider the shock driven into the ICM at the late SNR stage. In analogy with SNRs in galaxies, we assume that a fraction $\xi_{e}$ of the postshock thermal energy is given to the relativistic electrons. For a strong shock, the accelerated electron number is expected to be distributed with Lorentz factor $\gamma$ as $d N_{e} / d \gamma \propto \gamma^{-2}$ up to a maximum Lorentz factor $\gamma_{\max }$. The adiabatic compression of the magnetic field in X-ray clusters is expected to produce a magnetic field strength of $B \sim 10 B_{-5} \mu \mathrm{G}$ in the gas behind the forward-shock front. The accelerated electrons will emit synchrotron radiation at a frequency

$$
\nu=\gamma^{2}\left(\frac{e B}{2 \pi m_{e} c}\right)=0.3 B_{-5} \gamma_{4}^{2} \mathrm{GHz},
$$

where $\gamma_{4}=\left(\gamma / 10^{4}\right)$. The synchrotron cooling time of the electrons emitting at a frequency $\sim \nu$ is $t_{\text {syn }} \sim 2.5 \times 10^{7} \mathrm{yr}_{-5}^{2} \gamma_{4}^{-1}$. The synchrotron emission spans up to $\sim 2 \ln \gamma_{\max }$ decades in frequency, and the electrons carry an equal amount of energy per logarithmic Lorentz factor interval. Assuming that the ejecta does not decelerate significantly during the preceding CSM crossing, which holds for $\dot{M} \tau<M_{\mathrm{ej}}$, the synchrotron luminosity is therefore given by

$$
\nu L_{\nu} \sim\left(\frac{\xi_{e}}{2 \ln \gamma_{\max }}\right)\left[3\left(\frac{1}{6} v_{\mathrm{ej}}^{2}\right)\left(\frac{4 \pi}{3} R^{3} \rho_{\mathrm{ICM}}\right)\right] t_{\mathrm{syn}}^{-1} .
$$

Substituting $\gamma_{\max } \sim 10^{10}$ and $\xi_{e} \sim 0.05$, based on SNR observations (e.g., Dyer et al. 2001; Ellison et al. 2001; for a discussion see Keshet et al. 2003), we find that during the passage of 
the ejecta through the surrounding ICM, but before the ejecta starts to decelerate, the radio luminosity is given by

$$
\nu L_{\nu} \sim 6 \times 10^{32}\left(\frac{\nu}{1 \mathrm{GHz}}\right)^{1 / 2} n_{-3} v_{\mathrm{ej}, 3.5}^{5} t_{4}^{3} \mathrm{ergs} \mathrm{s}^{-1},
$$

where $t_{4}=\left(t / 10^{4} \mathrm{yr}\right)$. Equation (26) holds until the ejecta begins to decelerate, at

$$
t_{\mathrm{dec}} \simeq 10^{4}\left(\frac{M_{\mathrm{ej}, 0}}{n_{-3}}\right)^{1 / 3} v_{\mathrm{ej}, 3.5}^{-1} \mathrm{yr} .
$$

The luminosities given in equation (26) are consistent with those measured in galactic radio SNRs $\left(10^{32}-10^{34} \mathrm{ergs} \mathrm{s}^{-1}\right.$; e.g., Payne et al. 2004; Warren \& Hughes 2004), for which $n_{-3} \sim 10-1000$ and $t_{4} \sim 0.1-1$.

The continuum emission (accompanied by a subdominant inverse Compton component of upscattered microwave background photons) extends to high frequencies, following equation (26), up to optical frequencies, where the cooling time of electrons becomes comparable to the dynamical time. At higher frequencies the luminosity is frequency independent, $\nu L_{\nu} \propto \nu^{0}$. The peak luminosity is achieved at the deceleration time $t=$ $t_{\mathrm{dec}}$, when the flux and spectrum are given by

$\nu L_{\nu} \sim 1.3 \times 10^{36} M_{\mathrm{ej}, 0.6}^{2 / 3} v_{\mathrm{ej}, 3.5}^{3} n_{-3}^{1 / 3}\left(\frac{\nu / 10^{15} \mathrm{~Hz}}{n_{-3}^{2 / 3} v_{\mathrm{ej}, 3.5}^{2} M_{\mathrm{ej}, 0.6}^{-2 / 3}}\right)^{\alpha} \operatorname{ergs~s}^{-1}$,

where $\alpha=\frac{1}{2}$ for $\nu<10^{15} n_{-3}^{2 / 3} v_{\mathrm{ej}, 3.5}^{2} M_{\mathrm{ej}, 0.6}^{-2 / 3} \mathrm{~Hz}$, and $\alpha=0$ otherwise. This continuum emission can be searched for at optical and $\mathrm{X}$-ray wavelengths.

Looking back now to the expansion of the ejecta through the CSM, the emission of synchrotron radiation from the forward collisionless shock driven into the wind depends on the fraction $\xi_{B}$ of postshock thermal energy carried by the magnetic field. Radio $\mathrm{SNe}$ are commonly modeled assuming a near-equipartition magnetic field, $\xi_{B} \sim 0.1$ (e.g., Weiler et al. 1998). Under this assumption, the synchrotron emission during the CSM crossing phase is given by

$$
\begin{aligned}
\nu L_{\nu} \sim & 6 \times 10^{33} \operatorname{ergs~s}^{-1}\left(\frac{\nu}{1 \mathrm{GHz}}\right)^{1 / 2} \xi_{B,-1}^{3 / 4}\left(\frac{n_{-3} T_{* 1}}{v_{w, 1.5}^{2}}\right)^{7 / 4} \\
& \times v_{\mathrm{ej}, 3.5}^{13 / 2} t_{2}^{3} \times \begin{cases}1, & t>t_{\mathrm{rs}} \\
\left(\frac{R_{\mathrm{rs}} / v_{\mathrm{ej}}}{2 t}\right)^{7 / 2}\left[\ln \left(\frac{2 t_{\mathrm{ej}}}{t}\right)\right]^{3}, & t<t_{\mathrm{rs}}\end{cases}
\end{aligned}
$$

where $\xi_{B}=0.1 \xi_{B,-1}$ and $t=10^{2} t_{2}$ yr. The logarithmic factor cubed is due to the fact that part of the ejecta moves at a velocity $v$ faster than $v_{\mathrm{ej}}$. Although this factor formally diverges at small $t$, $v / v_{\mathrm{ej}}$ should not exceed a factor of 10 since the simulations for various models do not show shells moving at $v>10 v_{\text {ej }}$ (e.g., Höflich \& Khokhlov 1996; Dwarkadas \& Chevalier 1998). Since $v / v_{\mathrm{ej}} \gtrsim \ln \left(2 t_{\mathrm{ej}} / 2\right) \sim 10$ is obtained for $t \lesssim 1 \mathrm{yr}$, equation (29) is not applicable at earlier times. For the fiducial parameters, the $1 \mathrm{GHz}$ luminosity $\sim 1 \mathrm{yr}$ after the explosion is $\nu L_{\nu} \sim 1.4 \times$ $10^{35} \mathrm{ergs} \mathrm{s}^{-1}$. It should be kept in mind that at this stage, the luminosity depends sensitively on the velocity distribution in the ejecta; if the maximum velocity is, say, $5 v_{\mathrm{ej}}$, then the luminosity will be 10 times smaller.
Observationally, prompt or very early (a few years) radio emission from Type Ia SNe has not been detected. Sramek \& Weiler (1990; see Boffi \& Branch 1995) have presented upper limits on the radio fluxes for several events at distances of $\sim 20 \mathrm{Mpc}$, which translate to luminosity limits of $\nu L_{\nu} \lesssim 10^{35} \mathrm{ergs} \mathrm{s}^{-1}$. Three events were observed $\sim 2$ months after the explosion, but one event, SN 1981B, was observed near optical maximum. A $3 \sigma$ upper limit of $1 \mathrm{mJy}$ at $5 \mathrm{GHz}$ was also obtained a week before optical maximum by Eck et al. (1995) for SN 1986G in the nearby (4.2 Mpc; Tonry et al. 2001) galaxy NGC 5128 (Cen A), corresponding to $\nu L_{\nu}<1.1 \times 10^{35} \mathrm{ergs} \mathrm{s}^{-1}$. However, SN 1986G was a peculiar and underluminous SN Ia. These upper limits are comparable to the radio luminosities that we predict at an age of $\sim 1$ yr. Given the freedom in input parameters to equation (29), the dependence of its range of applicability on the speed of the fastest ejecta, and the small number of observed cases, this does not yet pose a serious discrepancy. However, tighter upper limits on the radio flux from additional SNe Ia would constitute independent evidence of the absence of a CSM. Similar conclusions were reached by Boffi \& Branch (1995).

\section{HAVE OPTICAL INTERGALACTIC SUPERNOVA REMNANTS BEEN DETECTED?}

In the course of narrowband imaging of the Virgo Cluster in search of intracluster planetary nebulae, Gerhard et al. (2002) have recently discovered a detached, spatially unresolved, emissionline object $\sim 17 \mathrm{kpc}$ in projection from the spiral galaxy NGC 4388. Optical spectroscopy of the object revealed line ratios, including [S II] $\lambda \lambda 6717,6731 / \mathrm{H} \alpha=0.1$, that are characteristic of $\mathrm{H}$ II regions. Based on this, Gerhard et al. (2002) classified this object as an intergalactic $\mathrm{H}$ II region in which the line emission is powered by one or two $\mathrm{O}$ stars. The massive stars are presumably members of a young cluster that was formed in situ in the ICM as a result of a recent collision between galaxies. The $\mathrm{H} \alpha$ equivalent width constrains the age of the star cluster to $\sim 3 \mathrm{Myr}$, too short for the stars to have formed in NGC 4388 and to have traversed the large distance, although the similar radial velocities of the galaxy and the emission-line region do suggest that the two are associated. The physical mechanism that could have led to intergalactic star formation is unclear, but perhaps the galaxy collision caused stripping and compression of gas along tidal tails, which then fragmented into stars when the gas was already unbound from its original galaxy. Gerhard et al. (2002) found a total of 17 candidate objects of this type in their narrowband data, with $\mathrm{H} \alpha+[\mathrm{N} \mathrm{II}]$ luminosities of order $10^{37} \mathrm{ergs} \mathrm{s}^{-1}$ (for an assumed Virgo distance of $17 \mathrm{Mpc}$; since the exact distances are unknown, the luminosities could be lower or higher by an order of magnitude).

Several (possibly related) objects have also been found by Ryan-Weber et al. (2004) in a narrowband imaging survey around nearby galaxies. Again, the emission-line objects are at large projected distances from their associated galaxies, but at similar velocities. Two of these candidates have been spectroscopically confirmed to be low-redshift objects based on the detection of both $\mathrm{H} \alpha$ and [O III] $\lambda 5007$ (the signal-to-noise ratio was too low for detection of additional lines). One object is $33 \mathrm{kpc}$ from the S0 galaxy NGC 1533 in the Doradus group (distance $21 \mathrm{Mpc}$ ), and another is $19 \mathrm{kpc}$ from the galaxies NGC 833 and NGC 835 in the compact group HCG 16 (distance $53 \mathrm{Mpc}$ ). The $\mathrm{H} \alpha$ luminosities and the deduced ages and numbers of ionizing stars are of the same order of magnitude as found by Gerhard et al. (2002) in Virgo.

While the intergalactic star formation option is a possibility, we point out that within current observational constraints, the 
emission-line objects could also be the remnants of the intracluster Type Ia SN population discovered by Gal-Yam et al. (2003), the observational signatures of which we have estimated above. The angular scale occupied by a remnant at the distance of the Virgo Cluster, $d=10 d_{1} \mathrm{Mpc}$, is

$$
\theta \sim \frac{R_{\max }}{d}=0.02\left(\frac{R_{18.5}}{d_{1}}\right),
$$

i.e., unresolved by ground-based optical telescopes but resolvable with the Hubble Space Telescope (HST) if $R_{18.5} \gtrsim 3$. The $\mathrm{H} \alpha$ luminosities of the putative intergalactic $\mathrm{H}$ II regions are similar to those of the more luminous SNRs in nearby galaxies (e.g., Blair \& Long 2004). Nearby SNRs of this luminosity are at least several thousand years old and are in their slow, radiative-shock phase, with strong low-ionization metal lines, in addition to Balmer lines. We estimated above that under typical conditions, intergalactic SNRs will not reach this phase. Furthermore, the object found by Gerhard et al. (2002) has detectable but relatively weak metal lines. However, considering the distance uncertainties (which lead to an order of magnitude uncertainty in the luminosity), stretching the parameters in equation (22), and allowing for line emission from dense clumps in the giant's wind, it cannot be excluded that this object is an intergalactic SNR. In the case of the objects studied by RyanWeber et al. (2004), the spectra have too low a signal-to-noise ratio to detect the metal lines, let alone perform the usual diagnostic tests distinguishing $\mathrm{H}$ II regions from SNRs.

In terms of numbers, Gerhard et al. (2002) estimate that there are $\sim 10^{3}$ similar emission-line objects in Virgo, but of order one-half, or even more, of these sources are likely background objects at high redshift (see Ryan-Weber et al. 2004). The measured Type Ia SN rate, per unit $B$-band stellar luminosity, in clusters and in elliptical galaxies (see summary in GalYam et al. 2002) is $R=0.2 \pm 0.1 h_{70}^{2} \mathrm{SNu}$, where $1 \mathrm{SNu}=$ SN century ${ }^{-1}\left(10^{10} L_{B, \odot}\right)^{-1}$, and $h_{70}$ is the Hubble parameter in units of $70 \mathrm{~km} \mathrm{~s}^{-1} \mathrm{Mpc}^{-1}$. The expected number of intergalactic SNRs in Virgo is therefore

$$
N_{\mathrm{SNR}} \sim R f L t \sim 150 R_{0.2} f_{0.2} L_{12.5} t_{3},
$$

where $R_{0.2}=R /(0.2 \mathrm{SNu}), f=0.2 f_{0.2}$ is the intergalactic stellar fraction, $L=3 \times 10^{12} L_{B, \odot} L_{12.5}$ is the total stellar $B$-band luminosity of Virgo (Sandage et al. 1985; Trentham \& Hodgkin 2002), and $t=10^{3} \mathrm{yr} t_{3}$ is the time during which a SNR emits $\mathrm{H} \alpha$ via nonradiative shocks that encounter the CSM. The number of emission-line objects is therefore also consistent, to an order of magnitude, with the SNR option, if the outer CSM radius is large enough to keep the SNR bright for about a thousand years.

Thus, if the intergalactic emission-line objects are SNRs, high angular resolution imaging should reveal resolved, shelllike morphologies with diameters of order $3 \mathrm{pc}$. If these objects are the remnants of the intergalactic Type Ia SN population, this would establish the fact that SNe Ia have a CSM at the time of explosion, with far-reaching implications for progenitor models.

We note that based on the rate parameters above, there should be an intergalactic SN Ia in Virgo about once per decade. However, such SNe may be missed because surveys for nearby SNe (e.g., Li et al. 2003) monitor individual galaxies, rather than the entire cluster. A candidate intergalactic Type Ia SN that went off in Virgo is SN 1980I (Smith 1981), which occurred in between three elliptical/S0 galaxies but was separated in projection by $\sim 50 \mathrm{kpc}$ from each. Twenty-five years after the explosion, the
SNR should be about halfway to its CSM reverse-shock crossing stage, with an $\mathrm{H} \alpha$ luminosity of $\sim 10^{34} \mathrm{ergs} \mathrm{s}^{-1}$, i.e., an $\mathrm{H} \alpha$ flux of $\sim 3 \times 10^{-19} \mathrm{ergs} \mathrm{s}^{-1} \mathrm{~cm}^{-2}$. Detection of such a weak line will be possible with the next generation of large $(\sim 30 \mathrm{~m})$ telescopes.

\section{INTERGALACTIC SUPERNOVA REMNANTS IN THE RADIO}

At the distance of the Virgo Cluster, $\sim 10 \mathrm{Mpc}$, the peak radio luminosities of intergalactic SNRs, lasting of order $10^{4} t_{4} \mathrm{yr}$, translate to fluxes of $\sim 0.1 \mathrm{mJy}$ at $\nu \sim 1 \mathrm{GHz}$. Considering the intergalactic SN rates above (eq. [31]), there should be of order $\sim 10^{3} t_{4}$ radio SNRs visible above this flux limit in Virgo, or about 10 SNRs per square degree. This is much smaller than the surface density of the background radio source population. A deep VLA survey of the Hubble Deep Field by Richards (2000) showed that the mean density of background radio sources brighter than $0.1 \mathrm{mJy}$ is about 3000 per square degree. He found that about one-half of the background sources were spatially extended, above the $\sim 2^{\prime \prime}$ resolution limit. Richards et al. (1998) found that the majority of the radio sources can be associated with luminous galaxies at redshifts $z \sim 0.1-1$, with mean $R$-band optical AB magnitudes of $\sim 22$, and generally brighter than 24.5. Star-forming disk galaxies and active galactic nuclei (generally in early-type galaxies) both contribute to the source counts at these fluxes, although the relative contribution of each class is unclear.

Intergalactic SNRs in Virgo would have an angular extent of order $1^{\prime \prime}$ and would not be associated with any background galaxy. Thus, by surveying $1 \mathrm{deg}^{2}$ in Virgo, to $0.1 \mathrm{mJy}$ in the radio and to $24.5 \mathrm{mag}$ in the optical, one could exclude most of the $\sim 3000$ background radio sources on the basis of their large sizes, association with distant galaxies, or both. Using follow-up radio observations with higher angular resolution of the remaining candidates, one could find of order 10 radio SNRs of intracluster $\mathrm{SNe}$ on the basis of their characteristic morphologies and sizes. A detection of this population, and measurements of its properties (SNR sizes, luminosities, spectra), would provide constraints on the intergalactic SN rate and on SN Ia progenitors.

Finally, from equation (29) the $1 \mathrm{GHz}$ continuum flux from the remnant of SN 1980I should be $\sim 0.25 \mathrm{mJy}$, which is detectable, although for some parameters the flux could be lower by an order of magnitude. With a radius of about 1 mas, the remnant should be unresolved.

\section{CONCLUSIONS}

We have estimated the properties of the remnants of Type Ia $\mathrm{SNe}$ that explode in the ICM. We have shown that if such SNe explode into a CSM, presumably produced by the wind from the donor giant star from which the white dwarf accreted mass, then intergalactic SNRs are no different from galactic SNRs, for an age of up to a thousand years. This conclusion is mostly unaffected by the large speed with which the SNR may travel through the ICM. Galactic and intergalactic SNe are distinct after they have expanded beyond the CSM radius. Since CSM and galaxy-ISM densities are comparable, galactic SNRs remain luminous emission-line sources in the ISM stage. Indeed, for any particular galactic SNR, it is difficult to determine observationally whether the ejecta is interacting with the CSM or the ISM, or even whether there ever was a CSM.

Intergalactic SNRs, by contrast, are optically luminous only during the CSM stage, if there is one. Once the ejecta reaches the ICM, the SNR quickly fades in the optical band. As a result, intergalactic SNRs can provide a unique test for the existence 
of a CSM around Type Ia SNe. If intracluster SNRs are found in the optical band, the corollary would be that Type Ia $\mathrm{SNe}$ have a CSM, supporting the progenitor model of accretion from a giant-star companion. Alternatively, a nondetection of these SNRs would imply the absence of a CSM. This would point to accretion from a main-sequence or subgiant companion, or to the "double degenerate" progenitor model, involving the merger of a white dwarf pair following the loss of orbital energy to gravitational radiation.

We have also shown that irrespective of the existence of a CSM, old intracluster SNRs are detectable in the radio band. Although identifying them among the more numerous background radio populations is challenging, one could take advantage of the low optical luminosity of SNRs in the ICM stage. In contrast to most of the background radio sources, which are associated with galaxies, the old intergalactic radio SNRs will have no associated optical source. Thus, a radio search could establish the existence of the old intracluster SNR population, and an optical search would provide valuable information on Type Ia SN physics.

Finally, we have speculated that several examples of compact intergalactic emission-line objects recently discovered in Virgo (Gerhard et al. 2002) and in two galaxy groups (Ryan-Weber et al.
2004) could be intergalactic SNRs rather than $\mathrm{H}$ II regions, as postulated before. We have argued that the luminosities and numbers of the newly discovered objects, although somewhat on the high side, are still roughly consistent with our estimates for SNRs. Consistency is possible given the simplifications inherent in our model, the allowed range of input parameters, and the uncertainties in the parameters describing the observed objects. High-resolution imaging with $H S T$ could potentially discriminate among the two options, based on the different morphologies of SNRs and H II regions. Deep pointed observations of the site of SN 1980I, a possible intergalactic SN Ia in Virgo, could test our predictions for the early-stage development of intergalactic SNRs.

We thank John Raymond and Jacco Vink for enlightening discussions on radiative processes in supernova remnants. This work was supported in part by NASA grant NAG5-13292 and by NSF grants AST 00-71019 and AST 02-04514 (for A. L.). A. L. is grateful for the kind hospitality of the Einstein Minerva Center at the Weizmann Institute of Science, where this work began.
Arnaboldi, M., et al. 1996, ApJ, 472, 145

Blair, W. P., \& Long, K. S. 2004, ApJS, 155, 101

Blandford, R. D., \& Eichler, D. 1987, Phys. Rep., 154, 1

Boffi, F. R., \& Branch, D. 1995, PASP, 107, 347

Branch, D., Livio, M., Yungelson, L. R., Boffi, F. R., \& Baron, E. 1995, PASP, 107, 1019

Calcáneo-Roldán, C., Moore, B., Bland-Hawthorn, J., Malin, D., \& Sadler, E. M. 2000, MNRAS, 314, 324

Chevalier, R. A., \& Raymond, J. C. 1978, ApJ, 225, L27

Ciardullo, R., Feldmeier, J. J., Krelove, K., Jacoby, G. H., \& Gronwall, C. 2002, ApJ, 566, 784

Ciardullo, R., Jacoby, G. H., Feldmeier, J. J., \& Bartlett, R. E. 1998, ApJ, 492, 62

Dubinski, J., Mihos, J. C., \& Hernquist, L. 1996, ApJ, 462, 576

Durrell, P. R., Ciardullo, R., Feldmeier, J. J., Jacoby, G. H., \& Sigurdsson, S. 2002, ApJ, 570, 119

Dwarkadas, V. V., \& Chevalier, R. A. 1998, ApJ, 497, 807

Dyer, K. K., Reynolds, S. P., Borkowski, K. J., Allen, G. E., \& Petre, R. 2001, ApJ, 551, 439

Eck, C. R., Cowan, J. J., Roberts, D. A., Boffi, F. R., \& Branch, D. 1995, ApJ, 451, L53

Ellison, D. C., Slane, P., \& Gaensler, B. M. 2001, ApJ, 563, 191

Feldmeier, J. J., Ciardullo, R., \& Jacoby, G. H. 1998, ApJ, 503, 109

Feldmeier, J. J., Mihos, J. C., Morrison, H. L., Rodney, S. A., \& Harding, P. 2002, ApJ, 575, 779

Ferguson, H. C., Tanvir, N. R., \& von Hippel, T. 1998, Nature, 391, 461

Gal-Yam, A., Maoz, D., Guhathakurta, P., \& Filippenko, A. V. 2003, AJ, 125, 1087

Gal-Yam, A., Maoz, D., \& Sharon, K. 2002, MNRAS, 332, 37

Gerhard, O., Arnaboldi, M., Freeman, K. C., \& Okamura, S. 2002, ApJ, 580, L121

Gregg, M. D., \& West, M. J. 1998, Nature, 396, 549

Hamuy, M., et al. 2003, Nature, 424, 651

Höflich, P., \& Khokhlov, A. 1996, ApJ, 457, 500

Keshet, U., Waxman, E., Loeb, A., Springel, V., \& Hernquist, L. 2003, ApJ, 585,128

Korchagin, V., Tsuchiya, T., \& Miyama, S. M. 2001, ApJ, 549, 244

Li, W., Filippenko, A. V., Chornock, R., \& Jha, S. 2003, PASP, 115, 844
REFERENCES

McCray, R., \& Wang, Z., eds. 1996, IAU Colloq. 145, Supernovae and Supernova Remnants (Cambridge: Cambridge Univ. Press)

Mendez, R. H., Guerrero, M. A., Freeman, K. C., Arnaboldi, M., Kudritzki, R. P., Hopp, U., Capaccioli, M., \& Ford, H. 1997, ApJ, 491, L23

Moore, B., Katz, N., Lake, G., Dressler, A., \& Oemler, A. 1996, Nature, 379, 613

Parker, E. N. 1963, Interplanetary Dynamical Processes (New York: Interscience)

Payne, J. L., Filipović, M. D., Pannuti, T. G., Jones, P. A., Duric, N., White, G. L., \& Carpano, S. 2004, A\&A, 425, 443

Raymond, J. 1991, PASP, 103, 781

Richards, E. A. 2000, ApJ, 533, 611

Richards, E. A., Kellermann, K. I., Fomalont, E. B., Windhorst, R. A., \& Partridge, R. B. 1998, AJ, 116, 1039

Ryan-Weber, E. V., et al. 2004, AJ, 127, 1431

Rybicki, G. B., \& Lightman, A. P. 1979, Radiative Processes in Astrophysics (New York: Wiley)

Sandage, A., Binggeli, B., \& Tammann, G. A. 1985, AJ, 90, 1759

Smith, H. A. 1981, AJ, 86, 998

Smith, R. C., Kirshner, R. P., Blair, W. P., \& Winkler, P. F. 1991, ApJ, 375, 652

Sramek, R. A., \& Weiler, K. W. 1990, in Supernovae, ed. A. G. Petschek (New York: Springer), 76

Theuns, T., \& Warren, S. J. 1997, MNRAS, 284, L11

Tonry, J. L., Dressler, A., Blakeslee, J. P., Ajhar, E. A., Fletcher, A. B., Luppino, G. A., Metzger, M. R., \& Moore, C. B. 2001, ApJ, 546, 681

Trentham, N., \& Hodgkin, S. 2002, MNRAS, 333, 423

Trentham, N., \& Mobasher, B. 1998, MNRAS, 293, 53

Truelove, J. K., \& McKee, C. F. 1999, ApJS, 120, 299

Tuohy, I. R., Dopita, M. A., Mathewson, D. S., Long, K. S., \& Helfand, D. J. 1982, ApJ, 261, 473

Vikhlinin, A. A., \& Markevitch, M. L. 2002, Astron. Lett., 28, 495

Warren, J. S., \& Hughes, J. P. 2004, ApJ, 608, 261

Weiler, K. N., et al. 1998, ApJ, 500, 51

Williams, B. F., Sjouwerman, L. O., Kong, A. K. H., Gelfand, J. D., Garcia, M. R., \& Murray, S. S. 2004, ApJ, 615, 720

Zibetti, S., White, S. D. M., Schneider, D. P., \& Brinkmann, J. 2005, MNRAS, 358,949

Zwicky, F. 1951, PASP, 63, 61 